\title{
Manejo quirúrgico de pseudoquiste pancreático gigante: Caso clínico
}

\author{
Italo Braghetto M. ${ }^{1,2}$, Rodrigo Jiménez Y. ${ }^{1}$, Owen Korn B. ${ }^{1}$ y Leonardo Arellano ${ }^{3}$
}

\section{Surgical management of giant pancreatic pseudocyst: Case report}

Introduction: Pancreatic pseudocysts (PQP) correspond to $80 \%$ of cystic lesions of the pancreas. A cystic tumor must be ruled out, which represents $10 \%$ to $15 \%$ of pancreatic cysts and $1 \%$ of malignant tumors, so the study and differential diagnosis is essential. The term giant pancreatic pseudocyst is traditionally used when the size is greater than $10 \mathrm{~cm}$. There are few cases of giant PQP in the national literature. Objective: To report a clinical case with giant $P Q P$, to analyze the differential diagnosis and therapeutic options. Materials and Method: Patient with progressive abdominal distension, weight loss and anorexia post episode of acute pancreatitis. Abdominal computed tomography and magnetic resonance imaging confirm a giant intra-abdominal cystic lesion. The patient was treated with an open Roux-en-Y pancreatic cystojejunostomy. Analysis of the content aspirated during surgery suggests PQP. The most relevant literature is reviewed for discussion. Results: Excellent postoperative clinical results, the histopathological study of the cyst wall, confirmed the diagnosis of pancreatic pseudocyst. At one year of follow-up, the patient remains asymptomatic. Discussion: The preoperative study is crucial to determine the differential diagnosis and rule out neoplastic or cystic parasitic lesions. Reported giant PSQs are rare and their surgical management will depend fundamentally on their size, anatomical relationships, and the experience of the treating team. Key words: pancreatic pseudocyst; diagnosis; treatment.

\section{Resumen}

Introducción: Los pseudoquistes pancreáticos (PQP) corresponden al 80\% de las lesiones quísticas del páncreas. Se debe descartar un tumor quístico, que representan el $10 \%$ al $15 \%$ de los quistes del páncreas y $1 \%$ de los tumores malignos por lo que es fundamental el estudio y diagnóstico diferencial. El término pseudoquiste pancreático gigante se usa tradicionalmente cuando el tamaño es mayor de $10 \mathrm{~cm}$. Hay pocos casos de PQP gigantes en la literatura nacional. Objetivo: Reportar caso clínico con PQP gigante, analizar el diagnóstico diferencial y las opciones terapéuticas. Materiales y Método: Paciente con distensión abdominal progresiva, pérdida de peso y anorexia, posepisodio de pancreatitis aguda. Tomografía computarizada abdominal y resonancia magnética confirman lesión quística gigante intraabdominal. El paciente fue tratado con una cistoyeyunostomía pancreática abierta en Y de Roux. El análisis del contenido aspirado durante la cirugía sugiere PQP. Para la discusión se revisa la literatura más relevante. Resultados: Excelente resultado clínico postoperatorio, el estudio histopatológico de la pared del quiste confirmó el diagnóstico de pseudoquiste pancreático. Al año de seguimiento, el paciente permanece asintomático. Discusión: El estudio preoperatorio es crucial para determinar el diagnóstico diferencial y descartar lesiones neoplásicas o parasitarias quísticas. Los PSQ gigantes reportados son poco frecuentes y su manejo quirúrgico dependerá fundamentalmente de su tamaño, de las relaciones anatómicas y de la experiencia del equipo tratante.

Palabras clave: pseudoquiste pancreático; diagnóstico; tratamiento.

\section{Introducción}

Los pseudoquistes pancreáticos (PSP) corresponden al $80 \%$ de las lesiones quísticas del páncreas y causan complicaciones en $7 \%-25 \%$ de los pacientes con pancreatitis o trauma pancreático. La primera etapa en su manejo es descartar un tumor quístico. Los estudios para el diagnóstico son la ecografía, la tomografía computarizada (TC), resonancia magnética (RM), la colangio-pancreatografía retrógrada endoscópica (CPRE), la punción guiada del quiste con el estudio del líquido y la biopsia de la pared. La
'Departamento de Cirugía, Hospital Clínico Universidad de Chile. Santiago, Chile. ${ }^{2}$ Clínica RedSalud Providencia. Santiago, Chile.

${ }^{3}$ Clínica Redsalud Providencia, Unidad de Anatomía Patológica. Santiago, Chile.

Recibido 2020-08-07 y aceptado 2020-09-07

Correspondencia a: Dr. Italo Braghetto M. ibraghet@hcuch.cl 
elección del tipo de tratamiento depende del tamaño del tumor, la localización y extensión, el riesgo quirúrgico, la experiencia del cirujano y los recursos institucionales ${ }^{1-7}$.

Los pseudoquistes pancreáticos (PQP) son definidos como colecciones líquidas encapsuladas,

Figura 1. Inspección: gran distensión abdominal.
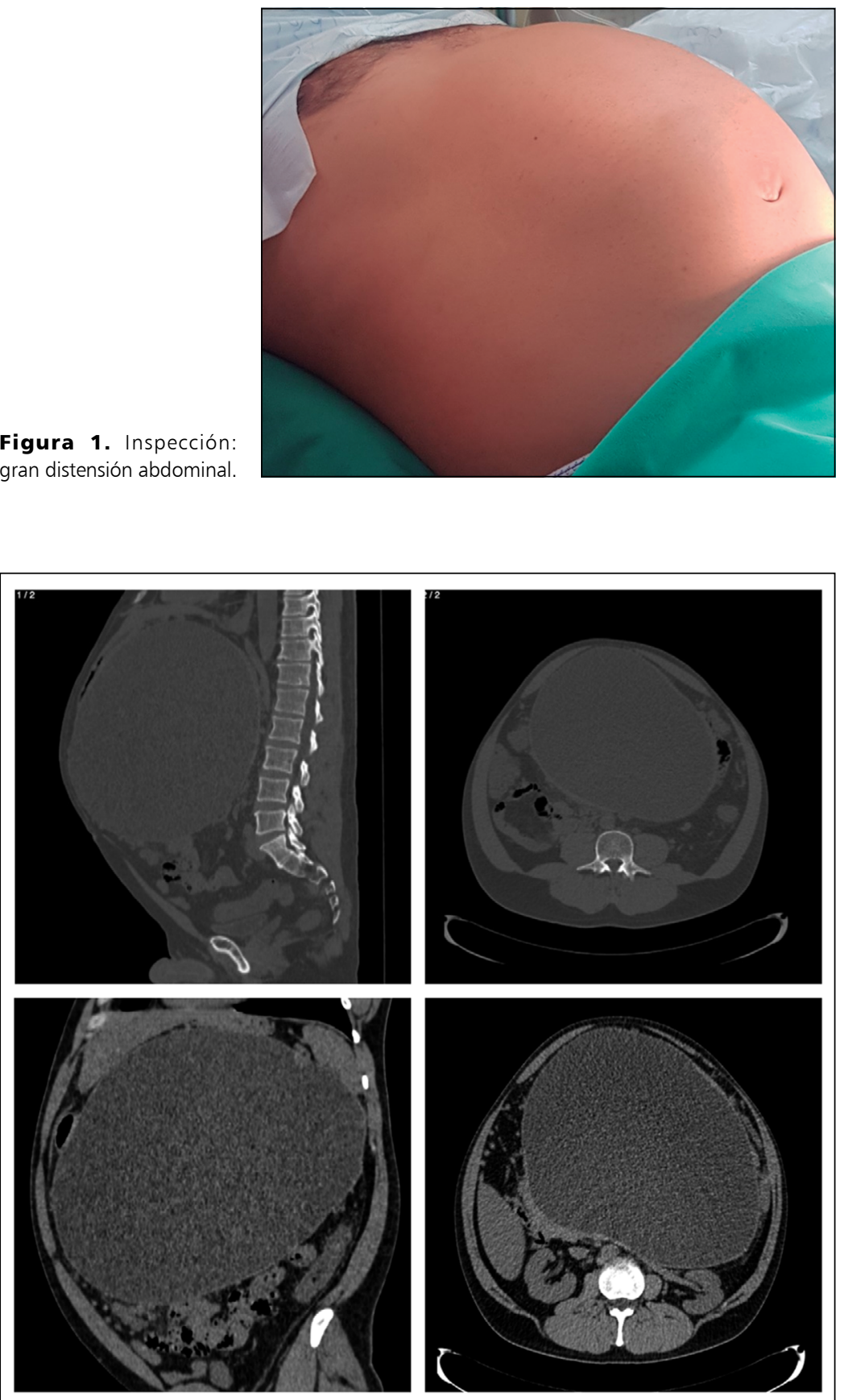

Figura 2. TC de Abdomen y pelvis: lesión quística gigante. usualmente extrapancreáticas, aunque pueden ser parcial o completamente intrapancreáticas ${ }^{7-11}$

Las opciones terapéuticas incluyen drenajes endoscópicos, percutáneos por radiología intervencional y drenaje quirúrgico, ya sea abierto, laparoscópico o robótico con distintas técnicas de reconstrucción ${ }^{11}$.

Se presenta caso clínico de paciente portador de un pseudoquiste pancreático gigante, uno de los más grandes reportados en la literatura internacional a propósito del cual se discute el diagnóstico diferencial y su enfrentamiento quirúrgico.

\section{Caso clínico}

Paciente de 41 años, sexo masculino baja de peso de $21 \mathrm{~kg}$ desde el inicio del cuadro clínico, con episodio de pancreatitis aguda moderada 1 año previo a consulta. Sin antecedentes de ingesta alcohólica ni otras causas de pancreatitis. En dicha hospitalización no se llegó a un diagnóstico causal claro, no reporta antecedentes quirúrgicos previos. Por cuadro de aumento progresivo de perímetro abdominal, anorexia, saciedad precoz, baja de peso progresivo sin dolor y gran distensión abdominal es derivado a cirugía (Figura 1).

\section{Exámenes de laboratorio}

Hematología: Hematocrito: 46,2\% Leucocitos: 7.500 plaquetas: 186.000 eosinófilos $2 \%$.

Perfil hepático: Bilirrubina Total: $0,41 \mathrm{mg} / \mathrm{dl}$, GGT: 62 U/1, GOT: 36 U/dl, GPT: 62 U/1 Fosfatasas alcalinas: $141 \mathrm{U} / 1$.

Perfil bioquímico normal, Creatininemia: 1,2 $\mathrm{mg} / \mathrm{dl}$

Marcadores tumorales: CEA: 3,6 ng/ml; Ca 19-9: $\mathrm{U} / \mathrm{ml} \mathrm{1;} \mathrm{alfafetoproteína:} \mathrm{2,4} \mathrm{ng/ml.}$

Serología hidatidosis negativa.

Imágenes: TC de abdomen y pelvis demostró extensa lesión quística de 27 x 21 x $26 \mathrm{~cm}$, de pared lisa, sin tabiques. Desplaza estómago hacia cefálico y contacta ampliamente el páncreas (Figura 2).

$\mathrm{RM}$ de abdomen y pelvis confirma lesión quística de $25 \times 20 \mathrm{~cm}$, paredes finas, sin tabiques. No se logra definir etiología sugiriendo la posibilidad de un pseudoquiste pancreático o quiste mesentérico (Figura 3).

Dado a la duda diagnóstica, el tamaño de la lesión y las posibles dificultades técnicas se prefirió la cirugía abierta.

En la exploración, se identifica gran lesión quística, se punciona y mide amilasa, la cual resulta $>$ 12.000 U/1. aspirando 8,5 1 de líquido (Figura 4). 
Mediante abordaje inframesocólico, resección de segmento de pared quística, biopsia rápida informa pared de pseudoquiste pancreático. Se realiza cistoyeyuno anastomosis en Y de Roux (Figuras 5, 6 y 7).

Por hallazgo de colelitiasis (cálculos pequeños) se practica colecistectomía.

Estudio histológico definitivo confirma la presencia de tejido conjuntivo denso con áreas fibrohialinas $(\mathrm{A})$ e infiltrado inflamatorio crónico focal $(\mathrm{B})$ concordante con pared de pseudoquiste pancreático (Figura 8).

El paciente presenta buena evolución posoperatoria, egresando al 3 día sin complicaciones.

Se realiza primer control 2 semanas post operatorio con excelente resultado clínico,

Seguimiento a 1 año está asintomático sin signos tomográficos de recidiva.

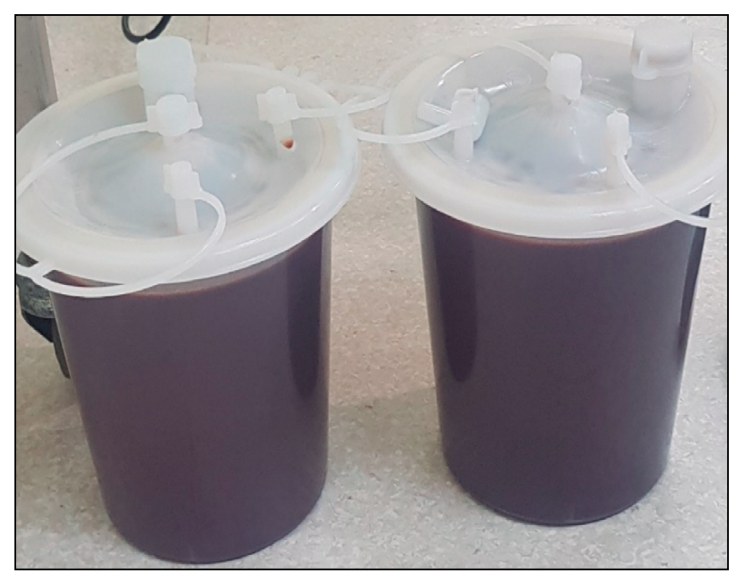

Figura 4. Contenido aspirado durante cirugía: 8,5 I (aprox.).

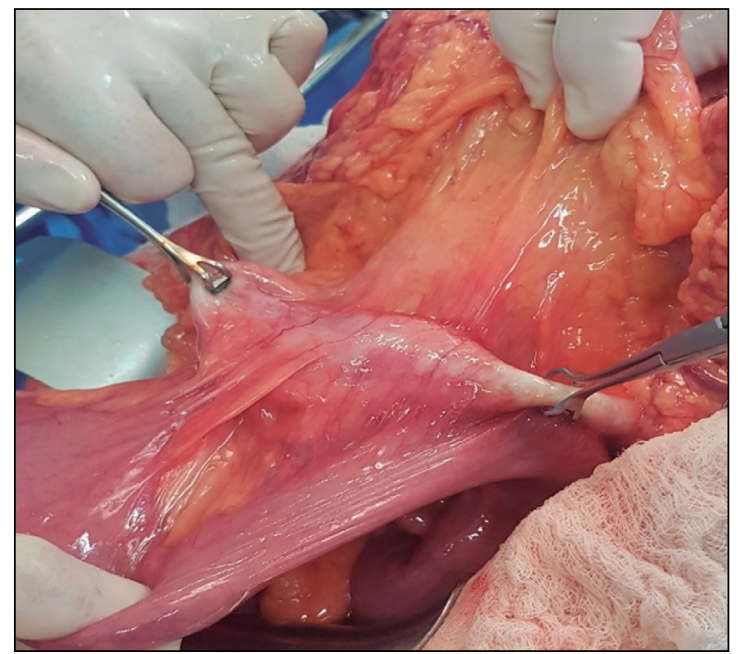

Figura 6. Pared de pseudoquiste pospunción previa a su apertura.

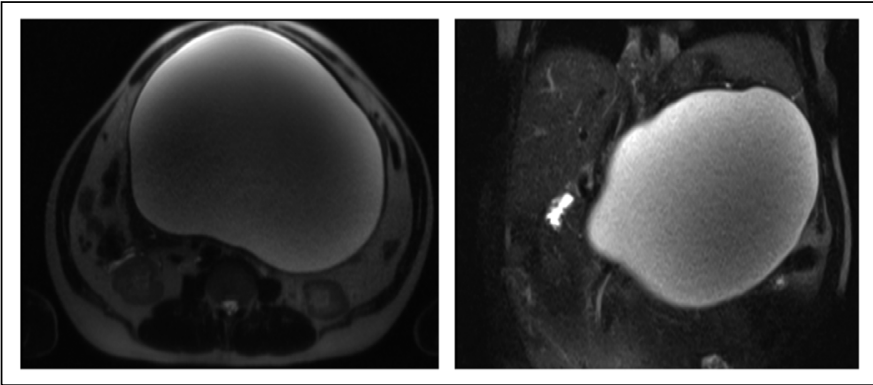

Figura 3. RM de abdomen y pelvis: confirma lesión quística gigante.

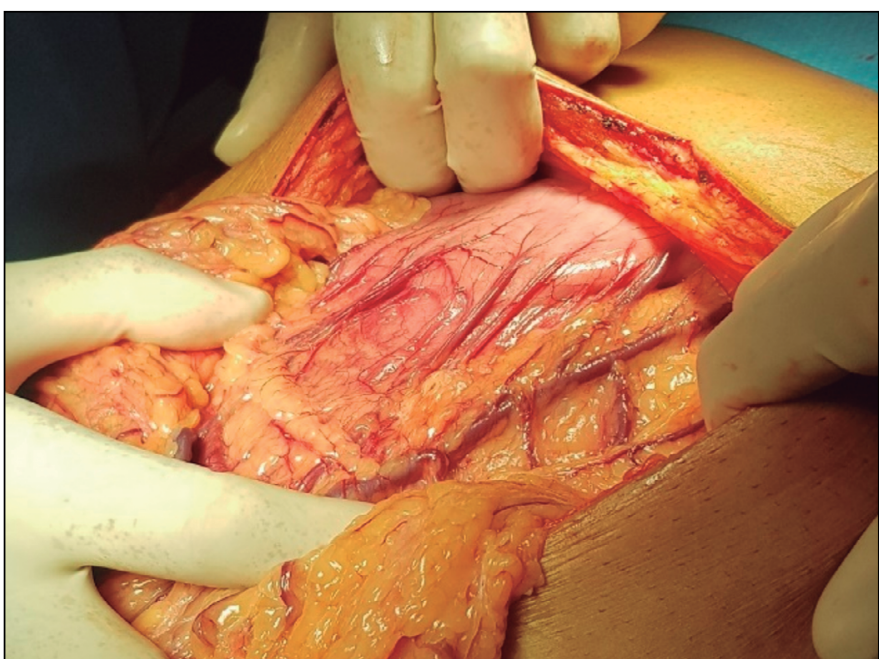

Figura 5. Exploración abdominal pospunción: estómago rechazado hacia anterior.

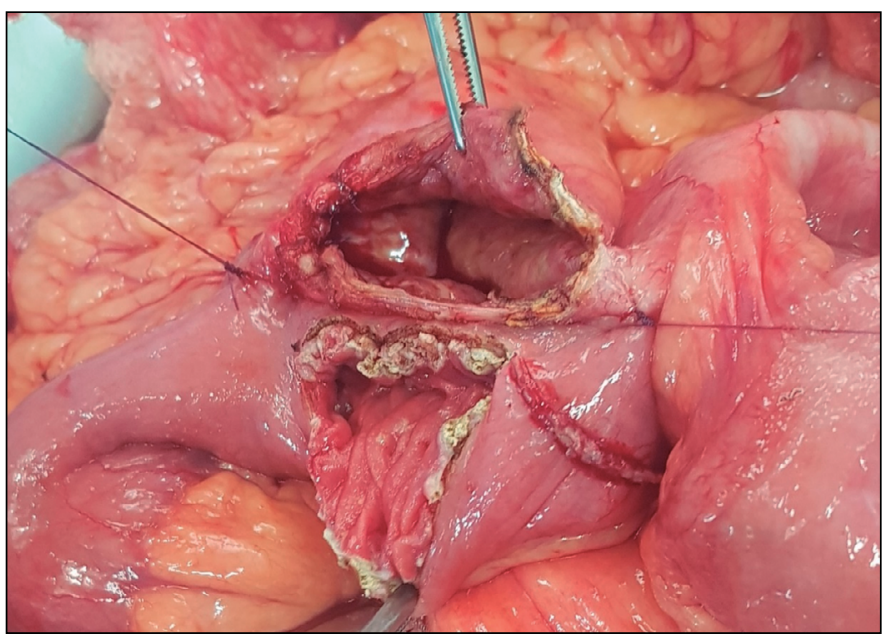

Figura 7. Apertura y resección parcial de pared de pseudoquiste. 


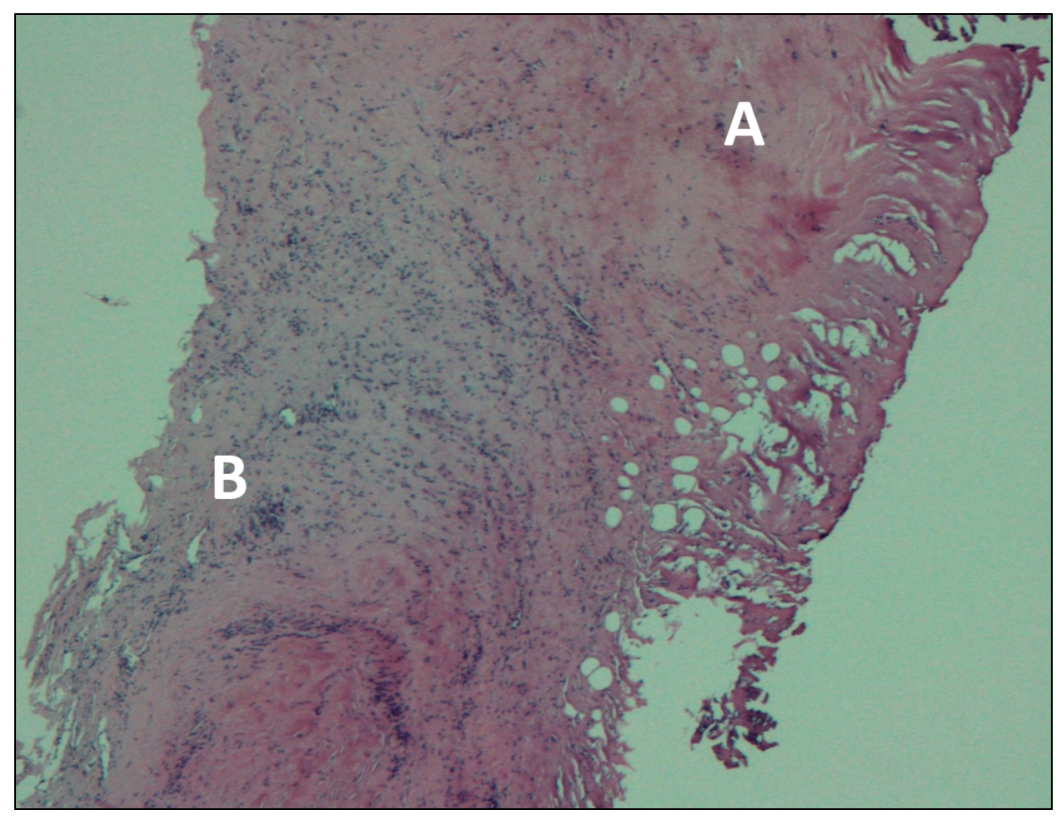

Figura 8. Estudio histológico de pared de pseudoquiste: tejido conjuntivo denso con áreas fibrohialinas (A) e infiltrado inflamatorio crónico focal (B) concordante con pared de pseudoquiste pancreático. muy pocos casos PQP gigante. Un PQP gigante (de 10 o más $\mathrm{cm}$ ) fue diagnosticado en 1882 , con aproximadamente 9,5 litros de contenido, tratado exitosamente con cirugía. Una revisión, recolectando 59 casos en 18 años, informó un pseudoquiste de $6,1 \operatorname{litros}^{1,5,10}$.

Como se desprende de esta revisión nuestro caso es uno de los más grandes reportados en la literatura.

PQP de mayor tamaño pueden provocar graves complicaciones como la compresión de órganos adyacentes (estómago, duodeno, conducto biliar común, conducto pancreático y vasos grandes), infección, hemorragia y formación de fístulas. Más del 50\% de los PQP gigantes desarrollan complicaciones que pueden ser potencialmente mortales ${ }^{11}$.

La elección del tratamiento de los PQP sigue siendo controversial. Las estrategias terapéuticas incluyen observación, drenaje endoscópico, drenaje percutáneo e intervenciones quirúrgicas. El abordaje quirúrgico es indicado cuando ocurren complicaciones o si se sospecha neoplasia quística ${ }^{11}$. Se recomienda seis semanas de observación después de la pancreatitis aguda como momento óptimo de la intervención quirúrgica para permitir la maduración de la pared del pseudoquiste de no haber evolucionado a la resolución espontánea. Varios factores influyen en el método de drenaje, incluyendo la edad, el tamaño y la ubicación del quiste. Las opciones quirúrgicas para el drenaje interno son cistogastrostomía, cistoduodenostomía y cistoyeyunostomía ${ }^{6,7,11,12}$.

La terapia endoscópica para los PQP, incluido el drenaje quístico-entérico, es una opción para los PQP que comprimen el lumen gástrico o yeyunal, que tiene un grosor de pared de menos de $1 \mathrm{~cm} \mathrm{y}$ la ausencia de estructuras vasculares mayores en la USE. El drenaje interno quirúrgico sigue siendo el estándar de oro y es el procedimiento de elección para PQP gigantes sintomáticos o complicados que tienen una pared madura ${ }^{11,12,14}$.

La literatura ha demostrado que un 20\%-50\% de los PQP menores a $6 \mathrm{~cm}$ y $27 \%$ de los que tienen $>10 \mathrm{~cm}$ de diámetro se resuelven espontáneamente y los que persisten más de 6 semanas o $>6 \mathrm{~cm}$ no se recuperan espontáneamente, a menudo presentan recurrencia, complicaciones, son sintomáticos y necesitan tratamiento quirúrgico ${ }^{19,20}$. Entre un $70 \%$ y $100 \%$ de $P Q P>10 \mathrm{~cm}$ requieren intervención abierta o endoscópica, o al menos drenaje percutáneo guiado por ecografía ${ }^{11-13}$.

El drenaje interno quirúrgico sigue siendo el estándar de oro. Un pequeño quiste en la cabeza puede ser susceptible de cistoduodenostomía endoscópica, pero se sugiere que para los PQP gigantes $(>15$ $\mathrm{cm})$, se prefiere una cistoyeyunostomía pues son 
predominantemente inframesocólicos ${ }^{11,13}$. Existen razones teóricas para preferir la cistoyeyunostomía a la cistogastrostomía:1) permite el drenaje independiente donde se encuentre el pseudoquiste, 2) las consecuencias de la dehiscencia anastomótica son menos graves, 3) la entrada de contenido gástrico en una cavidad de pseudoquiste agudamente inflamada puede provocar hemorragia. La cistoyeyunoanastomosis ofrece una alternativa más segura a expensas de una operación más larga, presenta una tasa ligeramente menor de complicaciones postoperatorias ${ }^{11-14}$.

Más recientemente algunas publicaciones reportan la técnica y los resultados con abordaje laparoscópico y robótico, pero la experiencia es escasa aún ${ }^{13,14}$.

El caso actual es uno de los más grandes reportados en la literatura tanto internacional como nacional ${ }^{15}$; se prefirió desde la partida un abordaje abierto ya que al analizar las imágenes preoperatorias la posibilidad de neumoperitoneo no era posible y la co- locación de los trocares podría haber sido riesgosa, de tal manera que desde la partida se abordó con una laparotomía media supra e infraumbilical de $15 \mathrm{~cm}$.

En conclusión, PSQ gigantes son raros y la literatura disponible sugiere como tratamiento un procedimiento quirúrgico de drenaje interno.

\section{Responsabilidades éticas}

Protección de personas y animales. Los autores declaran que para esta investigación no se han realizado experimentos en seres humanos ni en animales.

Confidencialidad de los datos. Los autores declaran que en este artículo no aparecen datos de pacientes.

Conflictos de interés: no hay.

\section{Bibliografía}

1. Lyngdoh TS, Konsam R, Marak Th B. Giant cystic lymphangioma of pancreas. ANZ J Surg. 2008;78:673-4.

2. Fujii M, Saito H, Yoshioka M, Shiode J. Rare Case of Pancreatic Cystic Lymphangioma. Intern Med. 2018;57:8137.

3. Rodrigues RV, Faias S, Fonseca R. Giant Pancreatic Cyst: An Unusual Entity. GE Port J Gastroenterol. 2016;23:314-5.

4. Testini M, Gurrado A, Lissidini A, Venezia P, Greco L, Piccinni G. Management of mucinous cystic neoplasms of the pancreas. World J Gastroenterol. 2010;16:5682-92.

5. Maranna H, Bains L, Lal P, Bhatia R, Yasir Beg M, Kumar P, Mallya V. Cystic Lymphangioma of the Greater Omentum: A Case of Partial Spontaneous Regression and Review of the Literature. Case Rep Surg. 2020;2020:8932017. Published online 2020 Jan 28. doi: $10.1155 / 2020 / 8932017$.
6. Brown SP, Williamson RC. Giant pancreatic pseudocysts: procedures in management. HPB Surg. 1992;6:129-31.

7. Behrns KE, Ben-David K. Surgical Therapy of Pancreatic Pseudocysts. Journal of Gastrointestinal Surgery 2008;12:2231-9.

8. Csendes A, Burdiles P, Csendes P, Albán M, Zamorano R. Quiste hidatídico pancreático. Reporte de un caso. Rev. Chil Cir. 2008;60:158-61.

9. Johnson LB, Rattner DW, Warshaw AL. The effect of size of giant pancreatic pseudocyst on the outcome of internal drainage procedures. Surg Gynecol Obstet. 1991;173:171-4.

10. Udeshika WAE, Herath HMMTB, Dassanayake SUB, Pahalagamage SP, Kulatunga A. A case report of giant pancreatic pseudocyst following acute pancreatitis: experience with endoscopic internal drainage. BMC Res Notes 2018;262. Published 2018 Apr 27. doi:10.1186/s13104-018-3375-9.

11. Matsuoka L, Alexopoulos SP. Surgical
Management of Pancreatic Pseudocysts. Gastrointestinal Endoscopy Clinics of North America 2018;28:131-41.

12. Wang, Y, Omar YA, Agrawal R, Gong Z. Comparison of treatment modalities in pancreatic pseudocyst: A population based study. World J Gastrointest Surg 2019;11:365-72.

13. Ånonsen $\mathrm{K}$, Sahakyan MA, Kleive D, Waage A, Verbeke C, Hauge T, et al. Trends in management and outcome of cystic pancreatic lesions - analysis of 322 cases undergoing surgical resection. Scandinavian Journal of Gastroenterology 2019;54:1051-7.

14. Doussot A, Perrin T, Rat P, Facy O. Robotic-assisted Roux-en-Y cystojejunostomy for pancreatic pseudocyst (with video). Journal of Visceral Surgery 2016;153:385-6. https:// doi.org/10.1016/j.jviscsurg.2016.06.005.

15. Alhassan S, Umar S, Lega M. One of the Largest Pancreatic Pseudocysts in the Literature: A Case Report. Cureus 2017;9:e1493. doi: 10.7759/cureus.1493. 